\title{
Computer Simulation of Thermally Sensitive Telechelic Star Polymers ${ }^{\dagger}$
}

\author{
Federica Lo Verso,* Christos N. Likos, and Hartmut Löwen \\ Institut für Theoretische Physik II: Weiche Materie, Heinrich-Heine-Universität Düsseldorf, \\ Universitätsstrasse 1, D-40225 Düsseldorf, Germany
}

Received: May 15, 2007; In Final Form: August 6, 2007

\begin{abstract}
Using molecular dynamics simulations, we study the conformational properties of thermally sensitive telechelic star polymers that carry attractive end groups. The telechelic star polymer functionality is varied between $f=1$ and $f=10$. As the temperature is lowered, we find a collapse from a "star burst" (sb) to a "watermelon" (wm) conformation and study the functionality- and polymerization-number dependence of the transition temperature. We also discuss partially collapsed configurations and multiple watermelon-like structures at higher functionalities.
\end{abstract}

\section{Introduction}

It is a challenging idea to fabricate smart materials with the desired rheological, electronic, and optical properties based on mesoscopically structured, self-organizing soft materials. ${ }^{1,2}$ Selfaggregating polymers with functionalized end groups are verypromising candidates to form network gel-like structures that sensitively respond to external perturbations, such as shear flow. In particular, polymers with a star-like architecture that are telechelic, that is, end-functionalized at the arms, have a potential to allow for complex aggregate formation in solution. The fundamental structural feature of a star polymer is its functionality, $f$, which counts the number of arms.

Recent progress in the synthesis of such telechelic star polymers has been achieved in various directions. Poly(Llactides) with a functionality, $f$, ranging between 2 and 6 have been prepared where a pyrene end group was added at every arm. ${ }^{3}$ The location of the pyrene end groups was measured for various functionalities, and a strong dependence on $f$ was observed. Other examples of synthesized telechelic star polymers are allyl-functionalized telechelic polystyrene, ${ }^{4}$ poly(ethyleneco-propylene) polymers bearing terminal self-complementary multiple hydrogen-bonding sites, ${ }^{5}$ and telechelic polylactones functionalized with trimethoxysilyl groups. ${ }^{6}$ Electrostatic selfassembly has also been exploited to form telechelic polyelectrolytes. $^{\text {? }}$

Furthermore, ABA-type triblock copolymers constitute good representatives of telechelic polymers with $f=2$ if the length of the A block is much smaller than that of the B block. Such ABA triblock copolymers comprising a relatively long poly(2(dimethylamino) ethyl methacrylate) and end-capped by short poly(methyl methacrylate) blocks were synthesized recently, ${ }^{8}$ although these systems are usually polydisperse. Controlled chemistry (anionic synthesis) leads for much-lower polydispersity and truly well-defined triblocks (e.g., ref 9). Different associates were found in aqueous solution at high dilution, namely, end-to-end linear associates and star-like "hairy" loose aggregates.

Generalizations toward higher functionalities are also conceivable by using many-arm blocks. For example, three-arm

\footnotetext{
† Part of the "Keith E. Gubbins Festschrift".

* Corresponding author. E-mail: federica@thphy.uni-duesseldorf.de
}

stars comprised of polyacetylene and polystyrene blocks were prepared, ${ }^{10}$ and block copolymer stars with a hydrophilic core and a hydrophobic corona are available. ${ }^{11,12}$ Low-functionality telechelics, which best match the model studied in this paper, are mono-, di-, and tri- $\omega$-zwitterionic, three-arm star symmetric polybutadienes. Experimental work has revealed that these selfassemble into different supramolecular structures. These structures include collapsed, soft-sphere conformations. ${ }^{13,14}$ In particular, static and dynamic light-scattering experiments have shown that samples with three zwitterion end groups present a low degree of interassociation between macromolecules, revealing a preference for intra-association. ${ }^{15,16}$ Further X-ray scattering and rheological experiments give evidence for the fact that this tendency persists at higher concentrations, all the way into the melt. ${ }^{13}$ There, the formation of transient gels has been found for the case of two- and three-zwitterion macromolecules, with the network characteristics depending on the molecular weight of the arms. ${ }^{14}$ Understanding the conformations of telechelic stars from first principles is therefore an important issue in polymer chemistry and physics.

The present paper deals with a monomer-resolved model for a single telechelic star polymer. The conformations of the star polymer are studied by molecular dynamics simulation. The essential parameters of the model are the molecular weight of the chains, that is, the number of monomers per arm, the functionality of the star, and the temperature that sets effectively the attraction strength between the functionalized endgroups. Complementary to this work, a theory for a low-functionalized telechelic star as well as for planar telechelic brushes has been proposed. ${ }^{17-20}$ Moreover, the formation of flower-like micelles with hydrophobic terminal groups that self-assemble in water has been explored by theory. Such aggregates exhibit a "bridging attraction"18,21 that can lead to a liquid-gas macrophase separation..$^{22} \mathrm{~A}$ previous account of our simulation data has been published elsewhere. ${ }^{17}$ Here we explore a wider functionality and number of monomers range.

The purpose of this paper is to complete the low-functionality study introduced in ref 17 and extend it to higher functionality, increasing at the same time the range of monomer per arm. In particular, in Section II we discuss our simulation method. In Section 3.1, we briefly resume the study for $f<6$ and we discuss more in detail the case of high number of monomers per arm. 
At a given, sufficiently low $T$, the wm configuration becomes more stable with respect to the sb one with decreasing $N$ and increasing $f$. In Section 3.2, we discuss stars with functionality up to $f=10$. Depending on the number of monomers per chain, the collapse from the starburst configuration toward aggregated structures here is more complicated and can involve "multiple watermelons"; that is, the end groups associate into different junction points and free dangling ends, and tadpole structures are also observed. Finally, in Section IV we draw our conclusions.

\section{Model}

A telechelic star polymer bears the possibility of attachment between different attractive terminal groups leading to intramolecular association. Clearly this is a competition between configurational entropy and attraction energy: at high temperature the association between different end groups will be suppressed, but at low-temperature it will be a typical configuration. In between, at intermediate temperature, there is a sharp crossover between these two structures. In previous work, ${ }^{17}$ we examined this conformational change for low functionalities up to 5. The corresponding aggregation typically consists of all end groups fused together such that the overall topology of the star polymer resembles that of a "watermelon". At higher temperatures, however, the polymer configuration possesses unfused end groups and can be called a "starburst" configuration.

In order to capture the various mechanisms in a simple model, we considered all monomers to be Lennard-Jones beads with tunable attractions acting among them. We studied isolated telechelic star-polymers with arm number, $f$, ranging in the interval $0<f<10$ and with a maximum of $N=200$ monomers per chain. To this purpose, we performed monomer-resolved molecular dynamics (MD) simulations. The equations of motion have been integrated using the so-called velocity form of the Verlet algorithm. ${ }^{23}$ In order to stabilize the temperature of the system, we applied a Langevin thermostat; ${ }^{23-25}$ that is, convenient random and friction forces are introduced related via the fluctuation dissipation theorem. According to the model described in refs 24-26, we consider three different contributions to the interaction between monomers. First, in order to mimic good solvent conditions, all nonterminal monomers interact via a truncated and shifted $\mathrm{LJ}$ potential

$$
V_{\mathrm{LJ}}(r)=\left\{\begin{array}{cc}
4 \epsilon_{\mathrm{LJ}}\left[\left(\frac{\sigma_{\mathrm{LJ}}}{r}\right)^{12}-\left(\frac{\sigma_{\mathrm{LJ}}}{r}\right)^{6}+\frac{1}{4}\right] & \text { for } r \leq 2^{1 / 6} \sigma_{\mathrm{LJ}} \\
0 & \text { for } r>2^{1 / 6} \sigma_{\mathrm{LJ}}
\end{array}\right.
$$

where $r$ is the separation between beads and $\sigma_{\mathrm{LJ}}$ and $\epsilon_{\mathrm{LJ}}$ set the length and energy scale, respectively. We define a dimensionless temperature $T^{*}=k_{\mathrm{B}} T / \epsilon_{\mathrm{LJ}}$ where $k_{\mathrm{B}}$ is Boltzmann's constant. Second, we modeled the chain connectivity employing the finite extension nonlinear elastic (FENE) potential: ${ }^{27}$

$$
V_{\mathrm{FENE}}(r)= \begin{cases}\frac{k_{\mathrm{FENE}}}{2}\left(\frac{R_{0}}{\sigma_{\mathrm{LJ}}}\right)^{2} \ln \left[1-\left(\frac{r}{R_{0}}\right)^{2}\right] & \text { for } r \leq R_{0} \\ \infty & \text { for } r>R_{0}\end{cases}
$$

Here $k_{\mathrm{FENE}}=20 \epsilon_{\mathrm{LJ}}$ and $R_{0}$ sets the maximum relative displacement between neighboring beads. For $R_{0}=1.5 \sigma_{\mathrm{LJ}}$ the total potential $V=V_{\mathrm{o}}+V_{\mathrm{FENE}}$ has a minimum at $r \sim 0.97 \sigma_{\mathrm{LJ}}$. Third, as described in ref 17 the end monomers of each chain interact via a full (attractive) Lennard-Jones potential. ${ }^{28}$

In a general model, where the introduction of a small hard core $R_{\mathrm{c}}$ of the central particles is necessary to accommodate a large number of arms, we need to add the interaction monomercore. This interaction is infinite for $r<R_{\mathrm{c}}$, whereas for $r>R_{\mathrm{c}}$ it is described by the same functional form used for the monomer-monomer interactions, for example, resulting from the combination of eqs 1 and 2 for monomers that belong to the first shell (nearest neighbor of the core-particle) and eq 1 , otherwise.

We perform standard MD simulations: the monomer mass, $m$, is assigned the value unity, defining thereby the characteristic time $\tau=\sqrt{m \sigma_{\mathrm{LJ}}^{2} / \epsilon_{\mathrm{LJ}}}$; the time step $\Delta t$ is in the range $\left[10^{-3} \tau, 10^{-2} \tau\right]$ with a total of $2 \times 10^{5}$ timesteps used for equilibration and 10 independent runs (when not specified) of $5 \times 10^{7}$ timesteps each to gather statistics.

\section{Simulation Results}

A. Low Functionality: Conformational Dependence on the Temperature and Chain Length. As introduced in ref 17, our study aims at establishing the relevant parameters driving different kinds of aggregation processes in telechelic starpolymer solutions. In the class of system we analyzed, there is a complex balance between various kinds of interactions (entropic, elastic, attractive) controlled by a large number of parameters that can have significant influences on the structure and dynamics of the system.

In this section, we focus on low-functionality star polymers $(f=2,3,4,5)$. Such stars are much easier to synthesize than high-functionality ones, much more common and, in fact, readily available experimentally. Moreover, a thorough understanding of the conformational properties of low-functionality telechelics is paramount to the understanding of those for high $f$-values because the latter are anticipated to often assume conformations in which a small subset of their arms collapse at their ends. As anticipated, the most-relevant experimental systems for our analysis are three- $\omega$-zwitterionic star symmetric polybutadienes in cyclohexane. ${ }^{13-16}$ By means of low-angle laser-light-scattering and dynamic-light-scattering experiments the authors of refs 13-16 studied three-arm stars with one, two, or three zwitterionic functional end groups, that is, terminal groups that associate in solution. Diluted samples with three zwitterionic end-capped chains, tend to interact intramolecularly, forming collapsed structures. In experiments as well as in our analysis, this possibility depends on the molecular weight and number of functional groups (we will return to this point later in the paper). Alternatively, high-concentration samples with two and three zwitterion end groups present a high degree of interassociation between macromolecules with the appearance of transient gels. Here we discuss on diluted systems in the limit in which intermolecular interactions can be neglected, in such a way to solve the intramolecular association of polymer chains and its effect on the star conformation.

One of the main parameters controlling the change in the star structure is the temperature: in Figure 1 we show, for $f=$ 3 and $N=10$, the possible molecular configurations. On the top, Figure 1a, we recognize the open-star configuration (starburst configuration), typical of common star polymers. In the range of temperature we analyzed $\left(0.01<T^{*}<1.2\right)$, the starburst configuration is stable for $0.2 \leqslant T^{*}<1.2$. After decreasing the temperature, we observed a finite probability to have doublets of attractive monomers, Figure 1b. This corresponds to a tadpole structure. Finally, for temperatures lower than 0.12 the stars collapse in the so-called watermelon structure, Figure 1c. The radius of gyration, $R_{\mathrm{g}}$, reflects the change in the molecular conformation: as described in our previous work we distinguish two different plateaus of $R_{\mathrm{g}}$ as a 


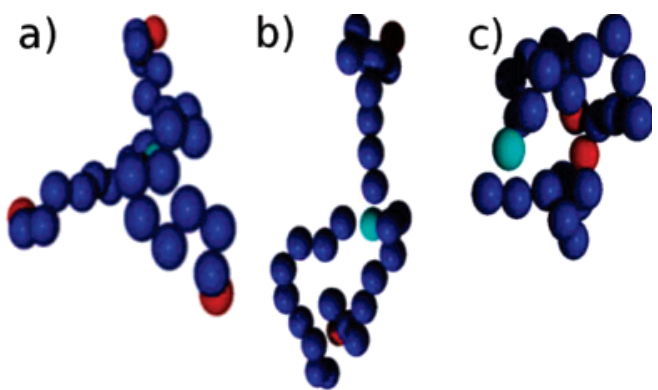

Figure 1. Intramolecular aggregation of a three-arm telechelic star (N=10): (a) $T^{*}=1.2$; (b) $T^{*}=0.2$; (c) $T^{*}=0.1$.

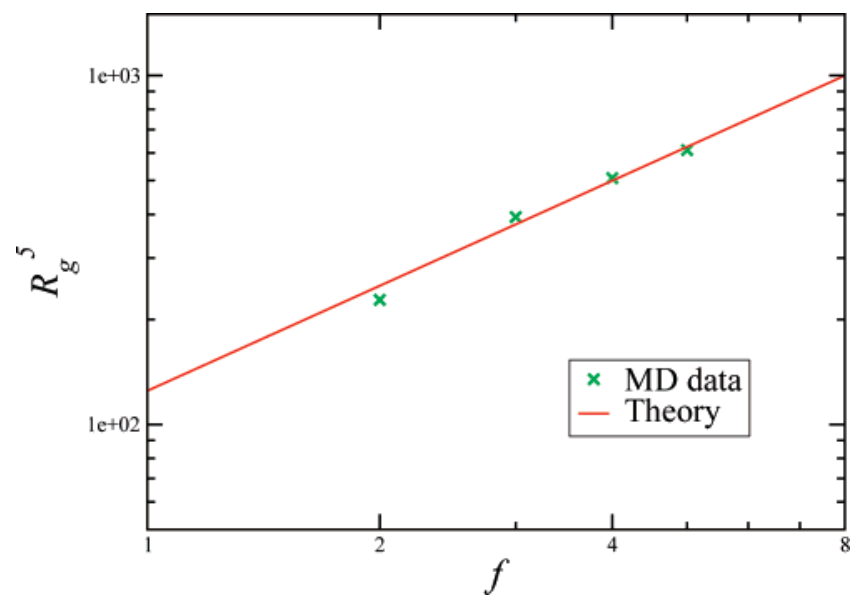

Figure 2. Double-logarithmic plot of the gyration radius, $R_{\mathrm{g}}$, of the starbust configuration for $T^{*}=1.2$ and $N=10$ against the functionality, $f$, demonstrating the $\sim f^{1 / 5}$ scaling of this quantity. The corresponding theoretical slope is shown by a straight line.

function of temperature that correspond to two almost constant $R_{\mathrm{g}}$ values. At high temperatures, our results agree with the scaling predictions on the basis of mean field arguments; ${ }^{29,33}$ for example, the sb radius of gyration is expected to scale with the arm number as $f^{1 / 5}$ (see, e.g., Figure $2, T^{*}=1.2$ and $N=$ $10)$.

For low temperature, a second plateau exists, which corresponds to the collapsed structure with a reduced radius. The trend is the same for the full range of $f$ although increasing $f$ the low-temperature plateau covers a wider $T^{*}$ range. We remark that for low functionality the additional entropic cost of bringing more chains into the watermelon is relatively small because there is a large amount of free space for the chains to move. This is more than balanced by the energetic gain of additional contacts between attractive monomers, which increase decreasing the temperature and increasing $f$.

In Figure 3, we show the comparison between the radius of gyration of the telechelic star with $f=3$ and $N=10,20,50$. Again we have the starbust plateau and the watermelon plateau: the temperature range of the latter decreases with $N$, indicating a lower $T^{*}$ to obtain the collapsed state. Moreover, we can notice how the error bar increases from the bottom to the top as a consequence of the lack of a unique/stable configuration.

In Figure 4, we show the instantaneous value of the attractive energy $\left(E_{\mathrm{t}}\right)$ for $f=3$ and $N=10,20$ and 50. Although for $N$ $=10$ and $T^{*}=0.12$ we have weak fluctuations of the energy around the value -3 , for $N=20$ and $N=50$ there are strong fluctuations and possibilities of intermediate configurations. To resume: increasing $N$ means that the transition temperature to the watermelon state drops: one needs the stronger energetic

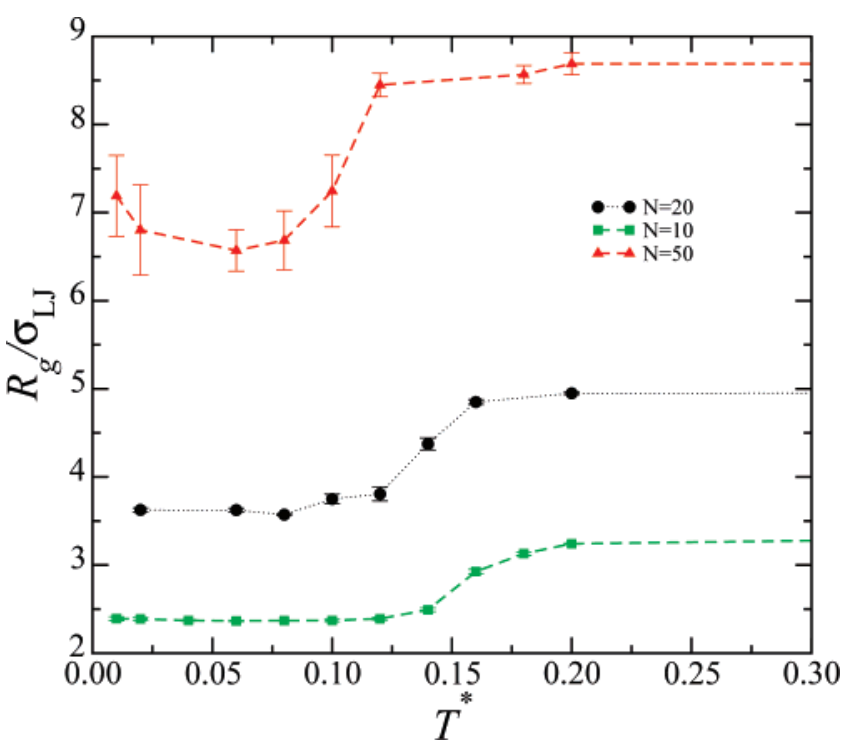

Figure 3. Radius of gyration vs temperature: $f=3$ and $N=10,20$, 50 .

gain to counterbalance the entropic loss. The trend with $N$ and $T^{*}$ is common to the full range of $f$ we consider in this section.

Figure 5 from ref 17 summarizes the parameter space we investigated corresponding to the open and collapsed structures: the main plot represents the simulation results. In the same reference, we clarified the interplay between the different contribution to the monomer-monomer interaction with a theoretical model. To resume: for fixed $N$ the wm configuration is more stable on increasing $f$ and lowering the temperature; this effect is due to the attractive energy contribution arising from the total number of contacts between terminal monomers, which becomes more and more important increasing the arm number and decreasing the temperature. Fixing $f$ and $T^{*}$, the wm configuration is stable below a certain value of $N$. Indeed the importance of the excluded volume interactions and the entropic contribution increase with the number of monomers. It is also to be expected that bond rigidity will disfavor the wm configuration. In this sense, however, the monomer scale appearing in our model can be interpreted as a Kuhn length, beyond which the bending rigidity of the chain renormalizes away to zero. We want to stress here that the range of $N$ in our simulations concerns the contact with experiments: large $N$ values in scattering experiments are indeed associated with the difficulties to obtain structural information, because the related $q$ range is inaccessible in X-ray (see ref 14). Increasing $N$ would bring the temperature leading to a collapse state at unphysically low values. To support our claims, we carried out simulations also for $N=100,200$. As shown in Figure 6, the watermelon configuration is indeed still possible for such high molecular weights but it occurs at very low temperature, $T^{*}=0.005$ for $N=100$ and $f=3$ and it came out only in a small fraction of runs. To make the analysis of the wm geometry more quantitative and to comply with our study, we calculated the instantaneous distance between the center of mass (CM), the grafting point (CORE), and the aggregation point (END), in conjunction with the radius of gyration.

The results are shown in Table 1: we can notice that the average distances turn out to be roughly equal to the watermelon $R_{\mathrm{g}}$. This gave us indications on the geometry of the collapsed configuration. Neither the grafting point nor the aggregation point is close to the center of mass. The CM-END distance is always smaller than the CM-CORE distance for trivial reasons: as $f$ end-monomers collapse on a single aggregation 


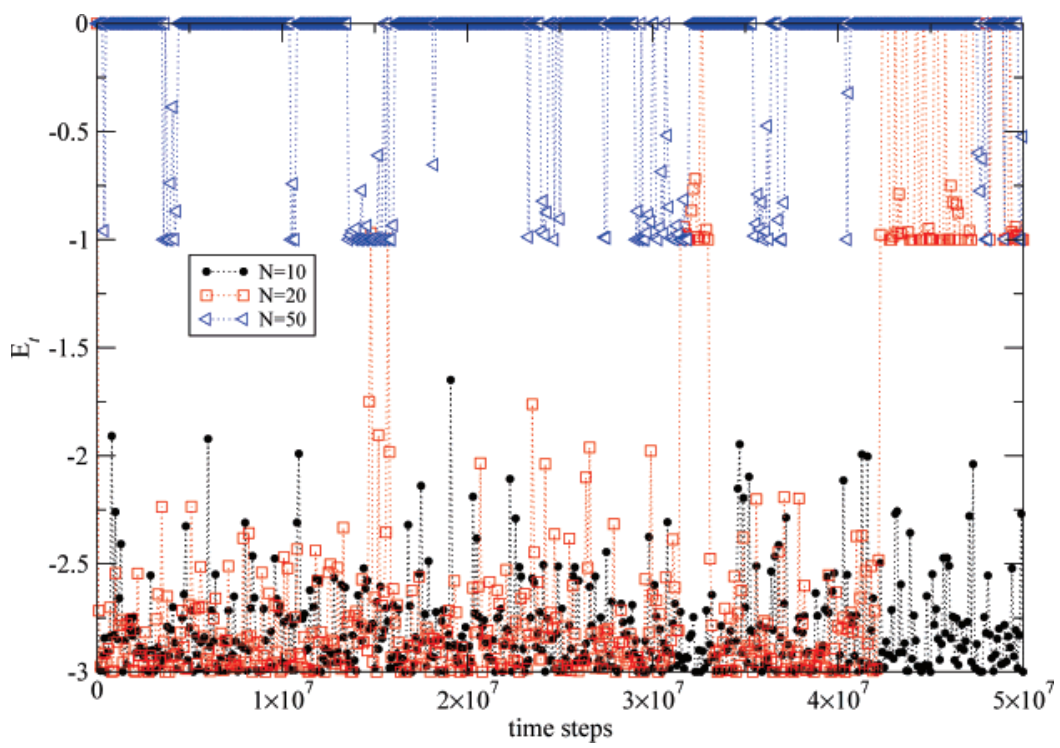

Figure 4. Instantaneous value of the attractive energy, $E_{\mathrm{t}}$, for $f=3$ telechelic stars at fixed temperature $T^{*}=0.12$, changing the number of monomers per arm.

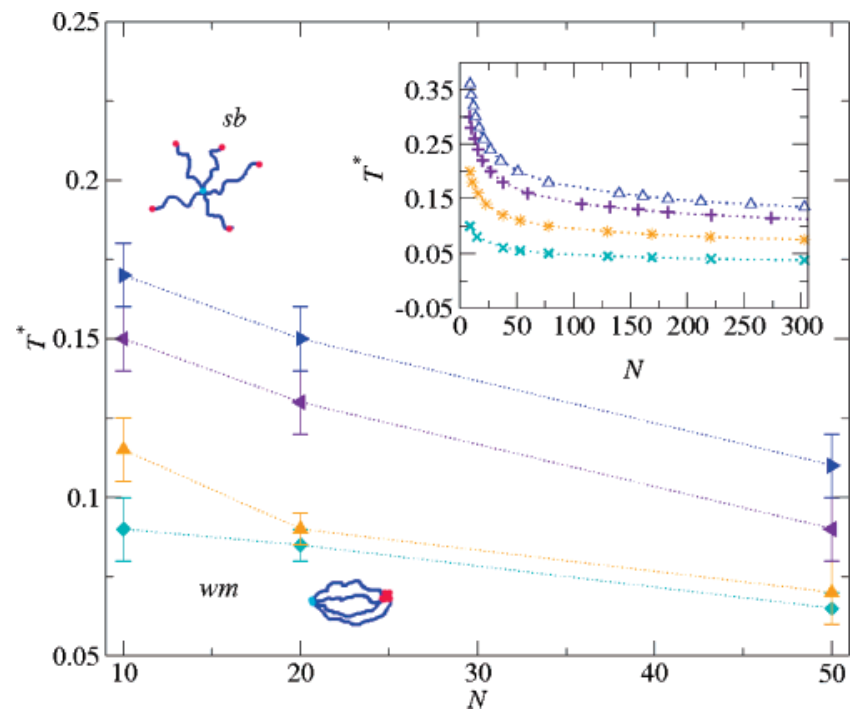

Figure 5. Regions of stability of the starburst and watermelon configurations vs the number of monomers per arm, from the top to the bottom $f=5,4,3,2$. The inset shows the theoretical prediction; see ref 17 .

point, they pull the $\mathrm{CM}$ to their side. This explains the fact that the trend is stronger as $f$ grows. At the same time, the discrepancy between the two distances diminishes with increasing $N$ because the relative weight of the $f$ end-monomers decreases accordingly.

B. High Functionality. In this section, we analyze telechelic stars with number of arms $f>5$; in particular, we changed $f$ between 6 and 10. At the beginning, we consider 10 monomers per arm trying to evidence the possible aggregation structures.

In Figure 7, we can observe the attractive energy distribution for $f=6$ : in the high-temperature regime $\left(0.4 \lesssim T^{*} \leq 1.2\right)$, the system assumes the starburst configuration $\left(E_{\mathrm{t}} \sim 0\right)$. Lowering the temperature, for example, $T^{*}=0.2$, we can have a rich variety of possible conformations with singlets, doublets, and/ or triplets of end monomers connected, as well as a few cases of 4 or 5 chain accumulation points, witnessed by the corresponding values of the attractive energy. For $T^{*}=0.18$, the value of the energy is still fluctuating between -8 and -6 , that is, a four-monomer wm plus a doublet or plus two free chains, and around -11 , that is, a five-monomer wm plus a free chain.

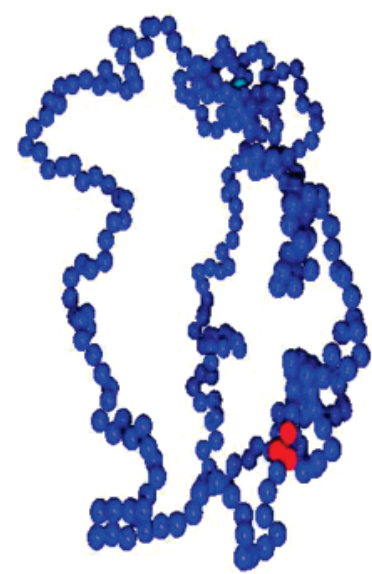

Figure 6. Watermelon configuration corresponding to $T^{*}=0.005, N$ $=100$, and $f=3$.

TABLE 1: Radius of Gyration, $\boldsymbol{R}_{\mathrm{g}}$, of Various Watermelon Structures Compared with the Distance between the Center of Mass and the Aggregation Point (CM-END) as well as the Distance between the Center of Mass and the Grafting Point (CM-CORE).

\begin{tabular}{lcccc}
\hline & $N$ & $R_{\mathrm{g}}$ & CM-END & CM-CORE \\
\hline$f=2$ & 10 & 2.192 & 1.987 & 2.481 \\
$T^{*}=0.04$ & 20 & 3.349 & 3.088 & 3.395 \\
$f=3$ & 10 & 2.366 & 1.795 & 2.180 \\
$T^{*}=0.06$ & 20 & 3.565 & 2.820 & 3.061 \\
$f=4, T^{*}=0.06$ & 10 & 2.474 & 1.693 & 2.007 \\
$f=4, T^{*}=0.04$ & 20 & 3.749 & 2.663 & 2.867
\end{tabular}

Finally, for $T^{*}=0.1$ all of the end monomers are close to each other giving rise to $E_{\mathrm{t}} \sim-12$. This value of energy is different from the one obtained considering the ideal, and indeed geometrically impossible case, where each couple of end monomers is in the minimum of the well potential; that is, $f(f-1) / 2=15$. We can gain some insight into the geometry of this aggregation by means of the position peaks in the radial distribution function, of the terminal monomers $g_{\mathrm{t}}(r)$, shown in Figure 8 . For $T^{*}=0.2$, the favorite interaction is the doublet (two terminal monomers connected) but the radial distribution function is broadly spread between 1 and 2, showing singlets, triplets, and so forth. By lowering the temperature (e.g., $T^{*}=$ 0.16 ), we can observe two additional peaks emerging. Finally, 


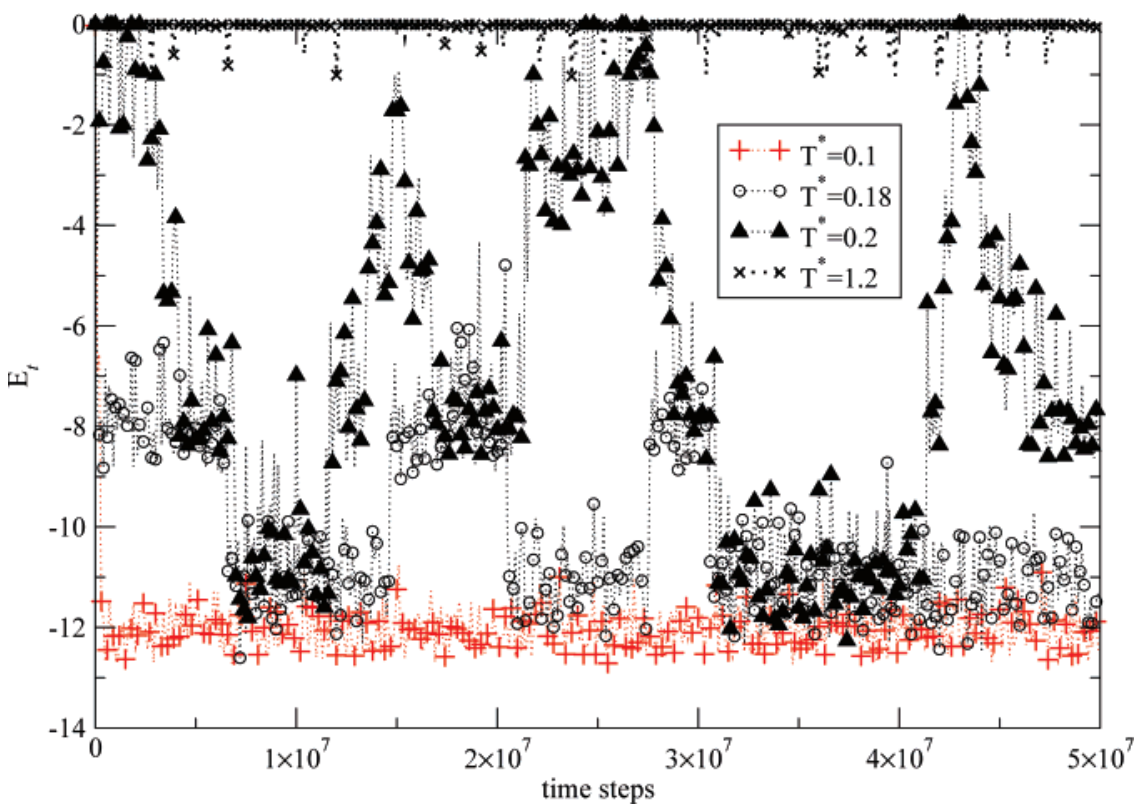

Figure 7. Attractive energy distribution for $f=6$ and $N=10$.

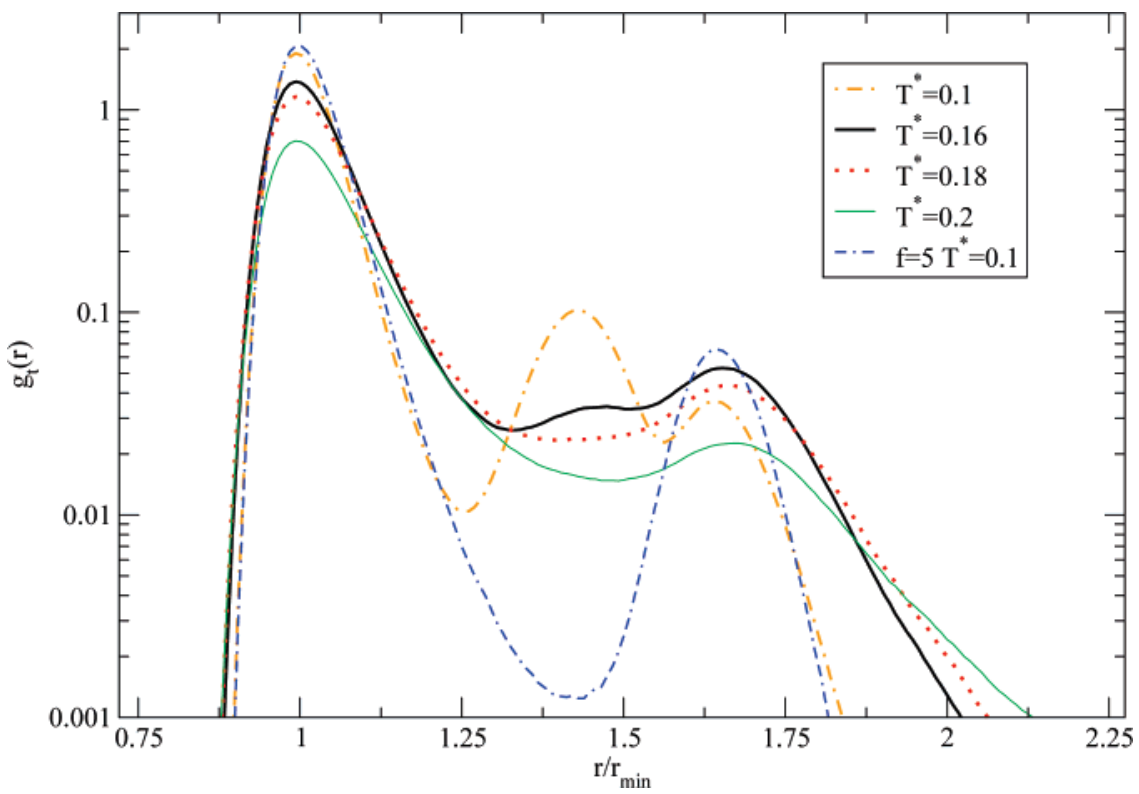

Figure 8. Radial distribution function of the terminal monomers changing the temperature. Here $f=6$.

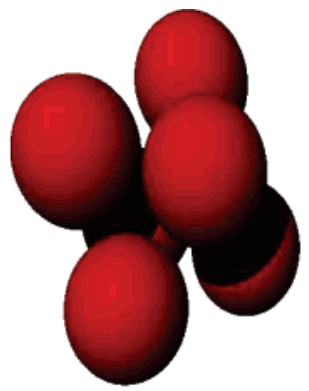

Figure 9. Aggregation geometry of end monomers for $f=6, N=10$, and $T^{*}=0.1$.

for $T^{*}=0.1$ all of the end monomers form an aggregate: from the radial distribution function we notice three peaks corresponding to $r / r_{\min } \sim 1, r / r_{\min } \sim 1.4$, and $r / r_{\min } \sim 1.7$, where $r_{\min }=2^{1 / 6}$. From the snapshot of the end monomers' aggregation (see red monomers in Figure 9) and from the above-mentioned analysis, we can draw two conclusions. First, the aggregation of red monomers seems to proceed progressively: at the beginning the system aggregate in a four-chain watermelon, finally the accumulation point consists of 5/6 chains. Second, we can derive the geometry of the end monomer aggregate: in the state in which they all aggregate, they form an octahedron that consists of two joined pentahedra sharing their square basis, see Figure 9. The second and third peak in the radial distribution function correspond to the distances between couple of end monomers, along the diagonal of the square, and at the vertexes of the octahedron respectively. For comparison, in Figure 8 we plotted the radial distribution function of the end groups $g_{\mathrm{t}}(r)$ for $f=5$ and $T^{*}=0.1$. In this case, as discussed in ref 17 , the geometrical distribution of end monomers covered a double tetrahedron sharing a triangular basis; the two length scales are related to the couples of monomers in the minimum of the well potential $(r \sim 1)$ and to the distance between the couple of terminal monomers at the vertexes of the tetrahedra.

The same analysis has also been performed for higher arm numbers. In Figure 10, we see the attractive energies corre- 


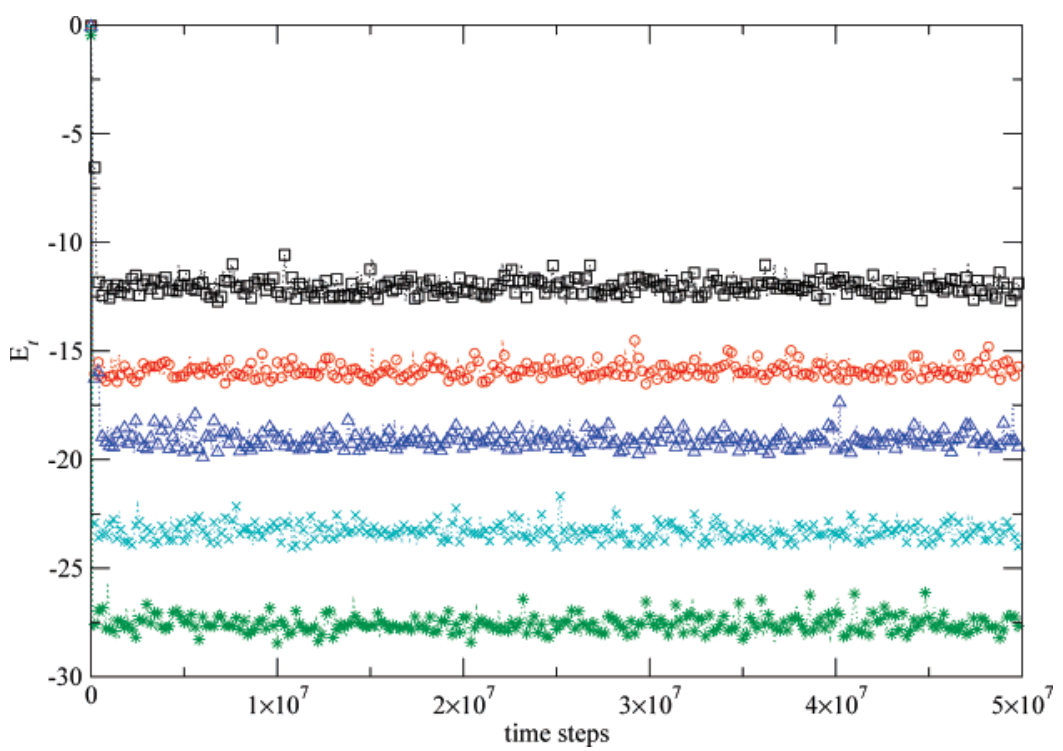

Figure 10. Attractive energy corresponding to collapsed configurations, $T^{*}=0.1, N=10$; from the top $f=6,7,8,9,10$.

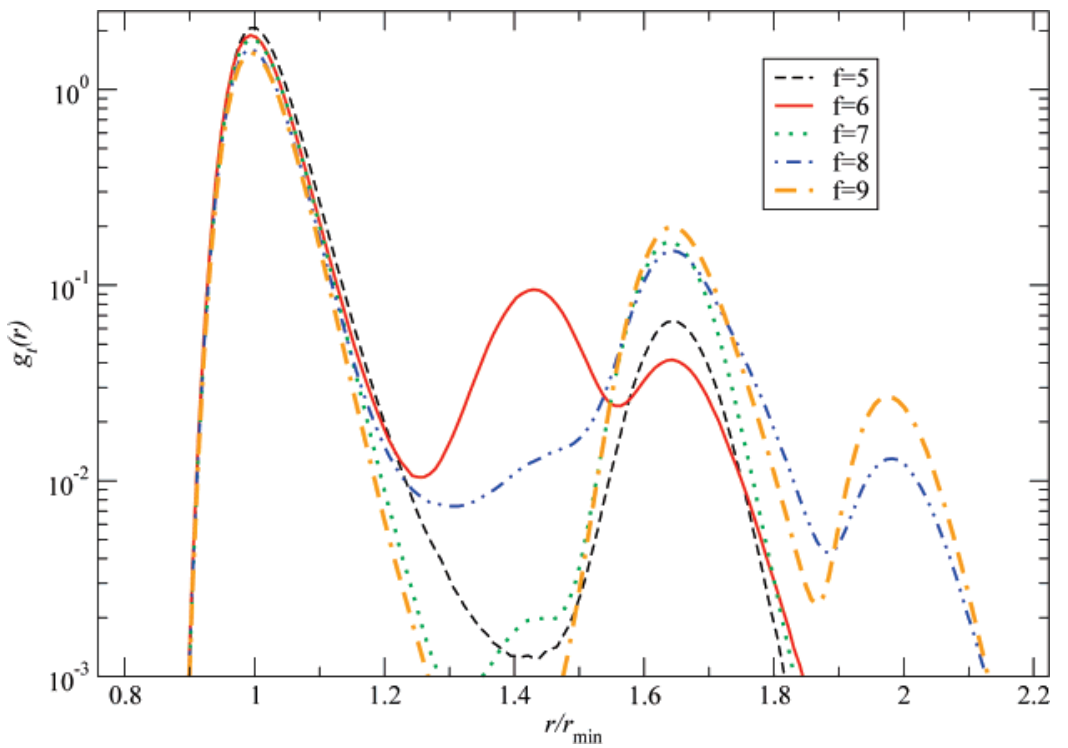

Figure 11. Radial distribution function of the attractive monomers, $T^{*}=0.1, N=10, f=5,6,7,8,9$.

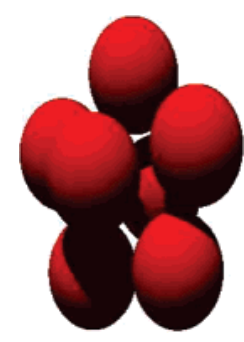

Figure 12. Snapshot of the end monomers aggregation for $f=7, T^{*}$ $=0.1, N=10$.

sponding to a collapsed state, for $N=10, T^{*}=0.1$, and $f=6$, $7,8,9,10$ : the values fluctuate around $-12,-15 / 16,-19$, -23 , and $-27 / 28$, respectively. In Figure 11, we plot the endmonomer radial distribution function for $f=5,6,7,8$, and 9 . For $f=7$, the snapshot in Figure 12 shows a configuration corresponding to a decahedron consisting of two joined hexahedra sharing their pentagonal faces: indeed, $g_{\mathrm{t}}(r)$ evidences two main peaks plus a small bump around $r / r_{\min }=1.5$, related to couples of end monomers at the diagonal of the pentagon. For $f=8,9$, at low temperature, the end monomers apparently

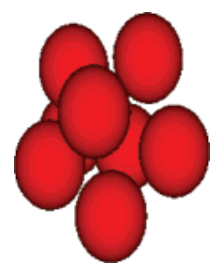

Figure 13. Snapshot of the terminal monomers aggregation for $f=8$, $T^{*}=0.1, N=10$.

do not form a regular polyhedron, we simply recognize some favorite configurations as in Figure 13, where some of red monomers form a pentagon that separates two of the remaining three particles on one side from the third one on the opposite side. This configuration corresponds to three different peaks in the radial distribution function. In particular, for $f=8$ and $f=$ 9 the third shell is considerably far away $\left(r / r_{\min } \sim 2\right)$. As a consequence, such next-nearest neighbors are not strongly attached to the nucleus and the aggregate is more fragile. The same conclusion can be deduced from the behavior of the radius of gyration as a function of the temperature. Up to $f=6,7, R_{\mathrm{g}}$ 

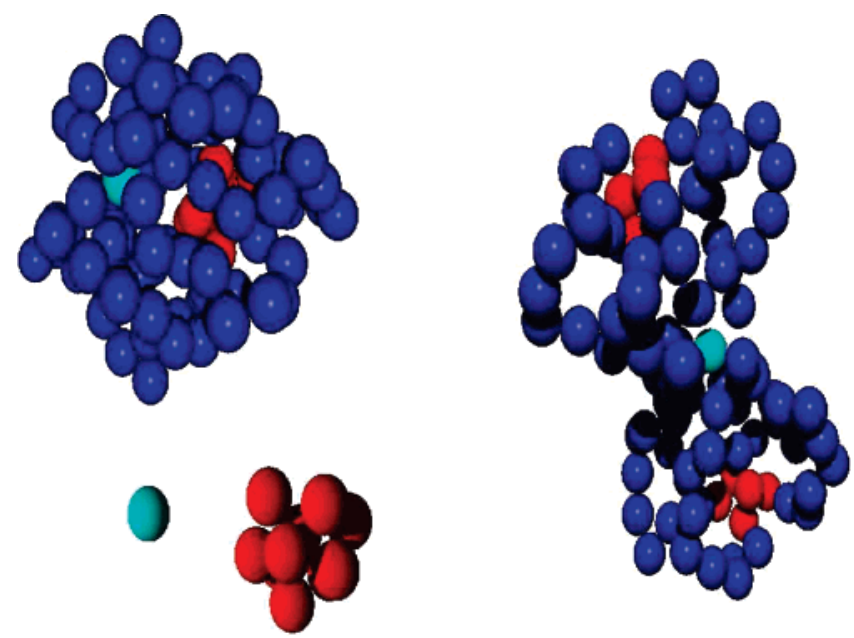

Figure 14. Ten end monomers aggregation for $N=10, T^{*}=0.1$

shows again the two plateaus, the low-temperature one corresponding to the collapsed state. By increasing $f(f=8,9,10)$, we find that the value of the radius fluctuates, giving a big error bar, which depends, for fixed temperature, on the lower probability of having a unique accumulation point. As an example, in Figure 14 we can observe two possible configurations, both corresponding to $T^{*}=0.1, f=10$ and $N=10$. On the left side (up), we have a collapsed structure, relative to $E_{\mathrm{t}}$ $=-27,28$; on the same side (bottom), we plotted the end monomers aggregate, which seems to form a hexagonal or pentagonal basis with three or four end monomers on one side. Moreover, on the right side we have a different configuration, with a double five-monomer aggregate: the system indeed formed a double watermelon. Finally, for $f=10$ we consider different molecular weights, $N=10,20,30$ to observe the change in the conformation due to the increase of the entropic penalty (see Figure 15). At high temperature, we found the starburst configuration (see, e.g., left-side snapshot). For lower temperatures (center- and right-side snapshots), as in the lowfunctionality case, the system presents a weaker tendency to the collapsed structure increasing the number of monomers per arm. As can be seen in Figure 15, the molecule exhibits instead a rich variety of intermediate structures. We conclude, thus, that although at functionalities $f \lesssim 10$ full collapses of telechelic stars on watermelon configurations are favored at low temperatures, for $f \geq 10$ the high entropic penalty arising from the accumulation of a large number of attractive monomers on a common aggregation site renders watermelons unstable. Intermediate configurations consisting of partial watermelons result instead.

\section{Conclusions}

The interplay between attraction and repulsion is a mechanism that can bring about enormous richness in phenomenology and allows for the control of the behavior of soft matter at various levels. Some very-well-known examples are the possibility to steer the disappearance of a liquid phase from the phase diagram of simple substances with spherosymmetric pair interactions ${ }^{34}$ and the ability to control the growth and size of finite, stable clusters in a colloidal suspension. ${ }^{35-43}$ In the aforementioned cases, the attraction and repulsion strengths and ranges pertain to the whole of a single, spherical, and structureless colloidal particle. Concomitantly, the effects of such changes, which can be induced by the introduction of nonadsorbing polymer or salt, express themselves on the macroscopic level, by influencing phase behavior and aggregation.

In our work, we have turned our attention to a different kind of interplay, namely, one that takes place at the monomer level of complex macromolecules with internal structures. We have established that by placing attractive end-monomers on lowfunctionality star polymers and controlling the attraction strength via temperature, one can induce quite-significant changes in the conformation of the molecule. A transition from a hollow to a starburst state has been seen to take place by increasing the temperature, and the critical value of the latter depends sensitively on functionality and degree of polymerization. Although our modeling was based exclusively on thermal systems, our results should also be applicable to ionic telechelics, for which the attraction strength can be tuned by changing the salt concentration and/or the solvent dielectric constant. Our finding of a reversible transition for these molecules also bears a similarity to the recently discovered light-induced opening of polyelectrolyte brushes ("nanoblossoms") for which the size and conformation is steered by regulation of the valency of the brush counterions. ${ }^{44}$

Concentrated solutions of telechelic stars are, therefore, verypromising candidates as tunable materials. Here, the intermolecular connectivity and phase behavior can be controlled by temperature, bringing about a variety of aggregation scenarios and allowing for external rheology control without affecting the chemistry of the substance. Work along the lines of investigating these properties by means of computer simulations is currently in progress. ${ }^{45}$

Acknowledgment. We thank Christian Mayer for helpful discussions. This work was supported by the Deutsche Forschungsgemeinschaft within the SFB-TR6 and by the Marie Curie European Network MRTN-CT-2003-504712.
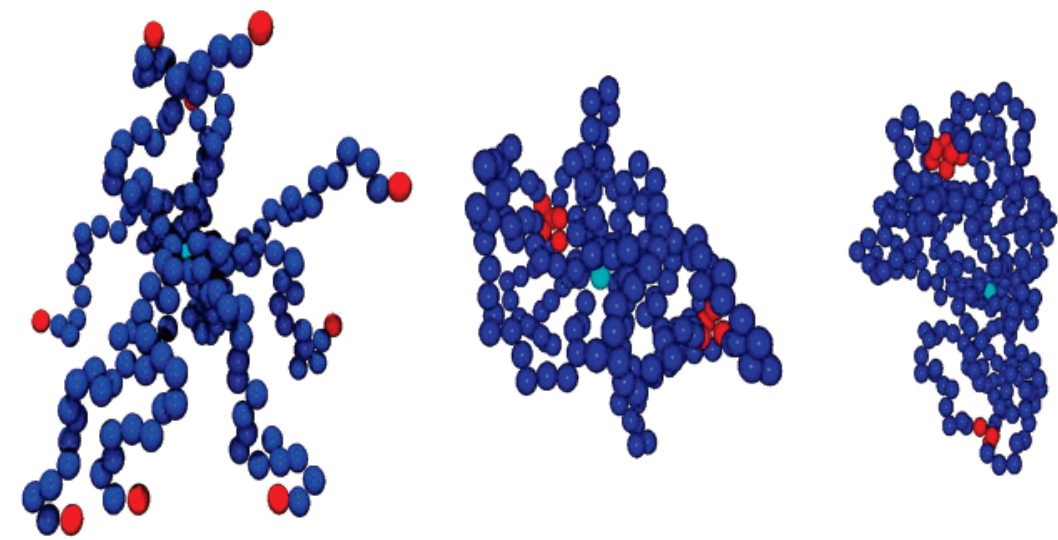

Figure 15. Ten red monomers aggregation for $f=10, N=20, T^{*}=1.2$ (left); $N=20$ and $T^{*}=0.1$ (center); $N=30$ and $T^{*}=0.1$ (right). 


\section{References and Notes} 225.

(1) Muthukumar, M.; Ober, C. K.; Thomas, E. L. Science 1997, 277,

(2) Theoretical Challenges in the Dynamics of Complex Fluids; McLeish, T., Ed.; Kluwer: London, 1997; NATO ASI Vol. 339.

(3) Danko, M.; Libiszowski, J.; Biela, T.; Wolszczak, M.; Duda, A. J. Polym. Sci., Part A: Polym. Chem. 2005, 19, 4586.

(4) Zhang, L. W.; Chen, Y. M. Polymer 2006, 47, 5259.

(5) Elkins, C. L.; Viswanathan, K.; Long, T. E. Macromolecules 2006 $39,3132$.

(6) Kricheldorf, H. R.; Thiessen, H. H. Polymer 2005, 46, 12103.

(7) Adachi, K.; Irie, H.; Sato, T.; Uchibori, A.; Shiozawa, M.; Tezuka, Y. Macromolecules 2005, 38, 10210 .

(8) Gotzamanis, G. T.; Tsitsilianis, C.; Hadjiyannakou, S. C.; Patrickios,

C. S.; Lupitskyy, R.; Minko, S. Macromolecules 2006, 39, 678.

(9) Koutalas, G.; Pispas, S.; Hadjichristidis, N. Eur. Phys. J. E. 2004, $15,457$.

(10) Sugiyama, K.; Karasawa, Y.; Higashihara, T.; Zhao, Y. L.; Hirao, A. Monatsh. Chem. 2006, 137, 869.

(11) Ganazzoli, F.; Kuznetsov, Yu. A.; Timoshenko, Macromol, E. G. Theory Simul. 2001, 10, 325.

(12) Connolly, R.; Timoshenko, E. G.; Kuznetsov, Yu. A. J. Chem. Phys. 2003, $119,8736$.

(13) Vlassopulos, D.; Pakula, T.; Fytas, G.; Pitsikalis, M.; Hadjichristidis, N. J. Chem. Phys. 1999, 111, 1760.

(14) Vlassopoulos, D.; Pitsikalis, M.; Hadjichristidis, N. Macromolecules

2000, 33, 9740

(15) Pitsikalis, M.; Hadjichristidis, N. Macromolecules 1995, 28, 3904.

(16) Pitsikalis, M.; Hadjichristidis, N.; Mays, J. W. Macromolecules 1996, 179,29

(17) Lo Verso, F.; Likos, C. N.; Mayer, C.; Löwen, H. Phys. Rev. Lett.

2006, 96,187802

(18) Semenov, A. N.; Nyrkova, I. A.; Khokhlov, A. R. Macromolecules 1995, 28, 1066 .

(19) Zilman, A. G.; Safran, S. A. Eur. Phys. J. E. 2001, 4, 467.

(20) Meng, X. X.; Russel, W. B. Macromolecules 2003, 36, 10112.

(21) Bhatia, S. R.; Russel, W. B. Macromolecules 2000, 33, 5713.

(22) Batra, U.; Pitsikalis, M.; Sioula, S.; Mays, J. W.; Huan, J. S.

Macromolecules 1997, 30, 6120.
(23) Allen, M. P.; Tildesley D. J. Computer Simulation of Liquids; Oxford University Press: Oxford, 1987.

(24) Grest, G. S.; Kremer, K. Phys. Rev. A. 1986, 33, 3628.

(25) Kremer, K.; Grest, G. S. J. Chem. Phys. 1990, 92, 5057.

(26) Jusufi, A.; Watzlawek, M.; Löwen, H. Macromolecules 1999, 32 4470 .

(27) (a) Grest, G. S.; Kremer, K.; Witten, T. A. Macromolecules 1987 20, 1376. (b) Grest, G. S. Macromolecules 1994, 27, 3493.

(28) See, for instance, Hansen J. P. and McDonald, I. R. Theory of Simple Liquids, 3rd ed.; Academic Press: London, 2006.

(29) Daoud, M.; Cotton, J. P. J. Phys. 1982, 43, 53

(30) Witten, T. A.; Pincus, P. A. Macromolecules 1986, 19, 2509.

(31) Witten, T. A.; Pincus, P. A.; Cates, M. S. Europhys. Lett. 1986, 2, 137.

(32) Likos, C. N.; Löwen, H.; Watzlawek, M.; Abbas, B.; Jucknischke, O.; Allgaier, J.; Richter, D. Phys. Rev. Lett. 1998, 80, 4450.

(33) de Gennes, P. G. In Solid State Physics; Ehrenreich, H., Seitz, F., Turnbull D., Liebert, L., Eds.; Academic: New York, 1978; Supplement 14: Liquid Crystals.

(34) See, e.g., Likos, C. N. Phys. Rep. 2001, 348, 267 and references therein.

(35) Sear, R.; Gelbart, W. M. J. Chem. Phys. 1999, 110, 4582.

(36) Sciortino, F.; Mossa, S.; Zaccarelli, E.; Tartaglia, P. Phys. Rev Lett. 2004, 93, 055701

(37) Mossa, S.; Sciortino, F.; Tartaglia, P.; Zaccarelli, E. Langmuir 2004 20, 10756.

(38) Campbell, A. I.; Anderson, V. J.; van Duijneveldt, J. S.; Bartlett, P. Phys. Rev. Lett. 2005, 94, 208301.

(39) Sanchez, R.; Bartlett, P. J. Phys.: Condens. Matter 2005, 17, S3351.

(40) Imperio, A.; Reatto, L. J. Phys.: Condens. Matter 2004, 16, S3769.

(41) Imperio, A.; Reatto, L. J. Chem. Phys. 2006, 124, 164712.

(42) Likos, C. N.; Mayer, C.; Stiakakis, E.; Petekidis, G. J. Phys.: Condens. Matter 2005, 17, S3363.

(43) Stiakakis, E.; Petekidis, G.; Vlassopoulos, D.; Likos, C. N.; Iatrou,

H.; Hadjichristidis, N.; and Roovers, J. Europhys. Lett. 2005, 72, 664.

(44) Plamper, F. A.; Walther, A.; Müller, A. H. E.; and Ballauff, M. Nano Lett. 2007, 7167.

(45) Lo Verso, F.; Likos, C. N.; Panagiotopoulos, A. Z. To be submitted for publication. 